\title{
STRUCTURAL AND KINEMATIC ANALYSIS OF LIFTING AND FOLDING MECHANISM OF MOBILE SHUTTER
}

Popa Lucretia $^{1}$, Stefan Moise Iulian ${ }^{2}$, Vasilica Stefan ${ }^{1}$, Liviu-Marian Ungureanu $^{3}$, Luciana Dudici ${ }^{3}$

${ }^{1}$ National Institute of Research-Development for Machines and Installations Designed to Agriculture and Food Industry, Romania; ${ }^{2}$ Etudes et Productions Schlumberger, France;

${ }^{3}$ University Politehnica of Bucharest, Romania

lucretia_popa@yahoo.com, stef_moise@yahoo.fr, valle_vali@yahoo.com, ungureanu.liviu.marian@gmail.com, luciana_ionita@yahoo.com

\begin{abstract}
The use of certain mechanisms in the construction of mobile platforms ensures that the products to be transported are loaded in the transport means without damaging them. This paper presents the structural and kinematic analysis of the lifting and folding mechanism of the mobile shutter of a commercial vehicle for transporting packed or containerised products. The structural analysis allows determining the structural groups (multipolar), which are the basis for realizing the mechanism for lifting and folding the shutter. The kinematic analysis consists in determining the kinematic parameters of all elements of the mechanism, the parameters underlying the kinetostatic calculation of the mechanism (determining the reactions in the kinematic couplings as well as the driving forces in the hydraulic cylinders). In order to highlight the kinematic parameters, the variation diagrams of the angular velocities and accelerations of the component elements were drawn. The kinematic analysis of the mechanism provides useful information regarding the lifting speed of the platform and, implicitly, the loaded material. A high-speed causes damage to the cargo loaded, and a small one causes a long loading time.The mathematical modeling of the mechanism was accomplished using Mathcad software. This mathematical modeling provides manufacturers with theoretical results that can be applied in practice for optimal sizing of the mechanism. The kinematic analysis made by simulation enables proper dimensioning of the elements of the mechanism, so that in the key positions the shutter is at the same level as the platform (in the maximum lifting position), or at the ground level, in the lower position, with the platform lowered for loading. This mechanism was actually used by INMA Bucharest to design and manufacture technological trailers for loading and transporting packed/containerised food products, trailers for transporting furniture, household appliances, and so on.
\end{abstract}

Keywords: structural analysis, kinematicanalysis,modular groups, diagrams.

\section{Introduction}

To facilitate loading of commercial vehicles with different products, the folding shutters (platforms) are used. These subassemblies are mounted at the rear of the machine and are usually driven hydraulically. The structure of these mechanisms is variable for the two operating phases (lifting the platform to the body level and folding the platform to the vertical position), making them easy to use to achieve the intended purpose.For kinematic analysis of the mechanism we used calculation procedures written by the authors in the MATLAB syntax. To create a clearer image how the mechanism works, the authors of the paper have also compiled animation computational procedures, and this animation has led to the theoretical data being confirmed. Velocity analysis involves determining "how fast" certain points on the links of a mechanism are traveling. Velocity is important because it associates the movement of a point ona mechanism with time. Often the timing in a machine iscritical.

There are known studies on a lot of mechanisms, but the study done in this paper is specific to the manufacture, being useful for this type of machines, which are implemented in the construction of the machines equipped with a lift-type shutter, designed for loading of the transported goods as well as the closure of the bins [1-3].

This study is the preliminary stage, necessary for solving the dynamic problem, to calculate the joint reaction and other dynamic characteristics, that are extremely important in a good dimensioning of the elements that compound the machine, reducing the unpleasant situation regarding the resistance of the construction. The kinetostatic and the dynamic analysis will be presented in the next papers.

All these analyses have been implemented in the design of the machine presented in Figure 1 [4].

This machine has been designed for loading/unloading and transport of packed/containerised products. 

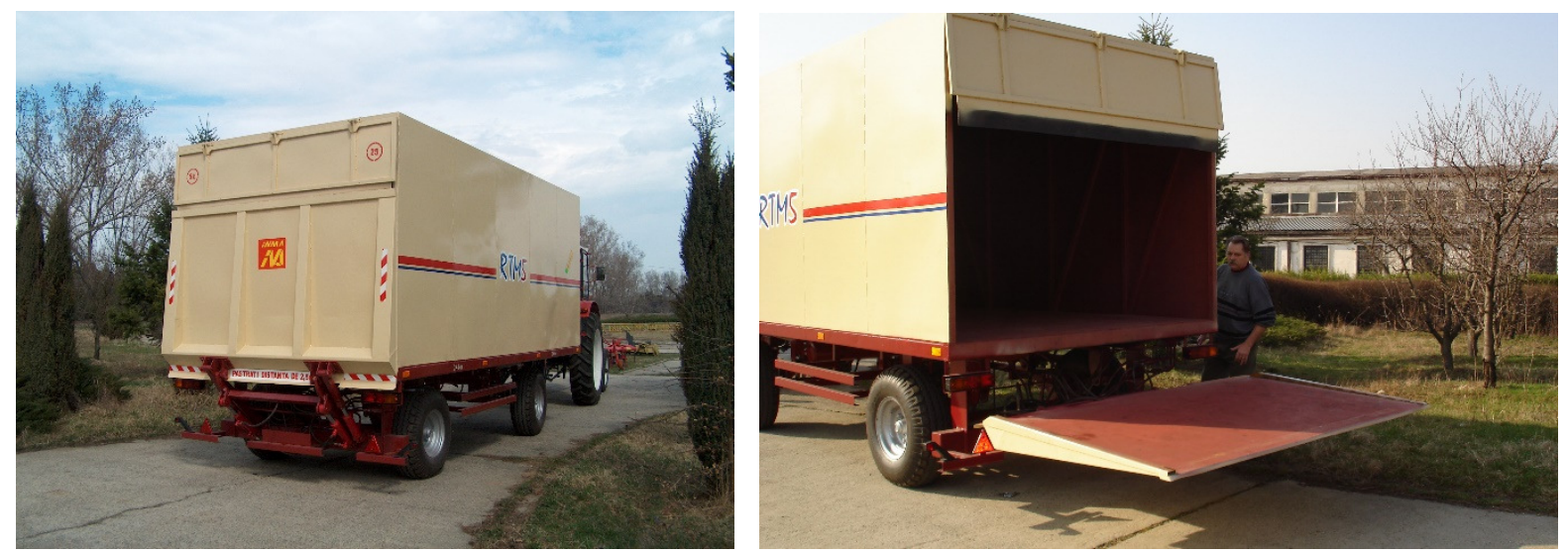

Fig. 1. Trailer with mobile shutter for transporting packed or containerised products made at INMA Bucharest - RTM5 [4]

\section{Materials and methods}

Figure 2 shows the kinematic diagram of the mechanism for lifting and folding the shutter of a commercial vehicle. As it can be seen in Figure 1, a platform $B T$, is rigidly mounted on the element 4 of the mechanism, on which the materials to be loaded into the machine are placed.

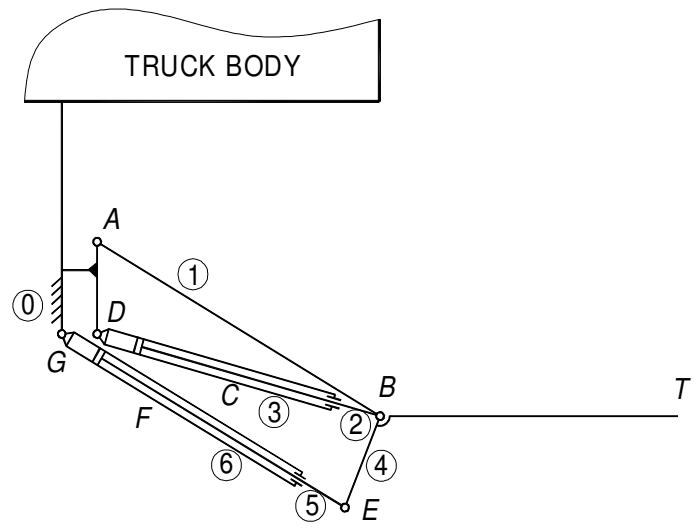

Fig. 2. Kinematic scheme of shutter lifting mechanism with $B T$ platform to lower position

In order to determine the kinematic parameters of the mechanism elements, the modular groups underlying the realization of the mechanism must be established, namely the structural analysis of the lifting and folding mechanism of the shutter must be made.

For the structural and kinematic analysis of the mechanism are known: the kinematic scheme of the mechanism, the dimensions of kinematic elements and the positions of the couplings adjacent to the base, the initial position of the mechanism, the relative linear velocities and accelerations in the $\mathrm{C}$ and D motor couplings, as well as the active strokes of the hydraulic cylinders. Once the structural groups have been established, the calculation program (in a known programming language) is drawn up to determine the kinematic parameters of the mechanism elements, the variation diagrams of the kinematic parameters are drawn and the results obtained are interpreted.

\section{Structural analysis of the mechanism}

The structural analysis of the mechanism, in the drawing in Figure 2, consists of: determining the kinematic couplings, determining the movable elements, determining the mobility degree of the mechanism, drawing up the structural scheme, establishing the structural groups, drawing up the multipolar scheme and writing the structural relation.

If the relative movements between the elements are taken into account, it can be noticed that the mechanism for lifting and folding the shutter (Figure 2) has the following lower couplings: $A(0 R 1)$, $B_{12}(1 R 2), C(2 T 3), D(3 R 0), B_{14}(1 R 4), E(4 R 5), F(5 T 6)$ and $G(6 R 0)$ [5-8]. 
The number of upper couplings is zero.

The movable elements of the mechanism are: $1\left(A, B_{12}, B_{14}\right), 2\left(B_{12}, C\right), 3(D, D), 4\left(B_{14}, E\right), 5(E, F)$, $6(F, G)$.

Given the number of movable elements and the number of kinematic couplings, results in the mobility degree of the mechanism, $M=2$, namely, two independent parameters are required so that the elements of the mechanism have uniquely determined movements. In this case, the structural scheme, the multipolar scheme and the structural relation are shown in Figure 3 [5, 8-15].

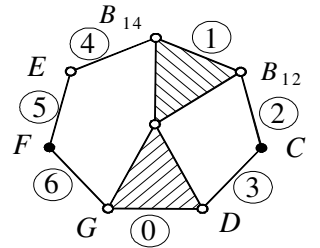

a)

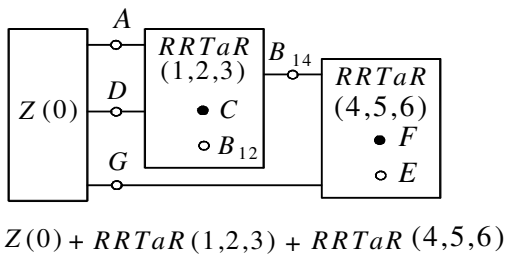

b)

Fig. 3. Structural scheme of mechanism in Figure 2 (a); multipolar scheme and structural relation (b)

Figure3shows that the mechanism consists of the base $Z(0)$, and motor dyadsRRTaR $(1,2,3)$ and $\operatorname{RRTaR}(4,5,6)$.

Further, the structural analysis of the mechanism in the motion phases is carried out.

In the first phase, the $B T$ platform of the mechanism is raised from the ground to the body of the trailer. In this situation, the platform must remain in the horizontal position to prevent objects from moving on the platform. In order to accomplish this, the element 4 , to which the $B T$ platform is attached, must be part of a parallelogram mechanism. In this regard, the hydraulic cylinder formed by the elements 5 and 6 is solidarized (blocked) to dimension $A B$. Since the $A G$ and $B E$ distances are equal, the $A B E G$ parallelogram is formed, which makes the $B T$ platform remain horizontal.

The kinematic scheme of the mechanism corresponding to this phase is given in Figure 4.

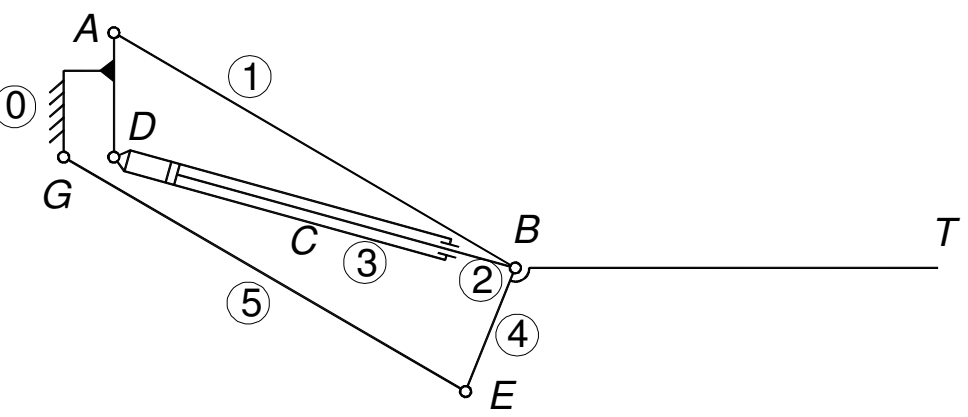

Fig. 4. Kinematic scheme of shutter lifting mechanism with cylinder 5-6 blocked at length $A B$

If the relative movements between the elements are taken into account, it is noted that the mechanism in Figure 4 has the following lower couplings: $A(0 R 1), B_{12}(1 R 2), C(2 T 3), D(3 R 0), B_{14}$ $(1 R 4), E(4 R 5)$ and $G(5 R 0)$. The number of upper couplings is zero.

The movable elements of the mechanism are: $1\left(A, B_{12}, B_{14}\right), 2\left(B_{12}, C\right), 3(C, D), 4\left(B_{14} E\right), 5(E, G)$.

Given the number of movable elements and the number of kinematic couplings, results in the mobility degree of the mechanism, $M=1$, namely, only one independent parameter is required so that the elements of the mechanism have uniquely determined movements.

Figure 5 shows the structural scheme, the multipolar scheme and the structural relation of the mechanism in Figure 4. 


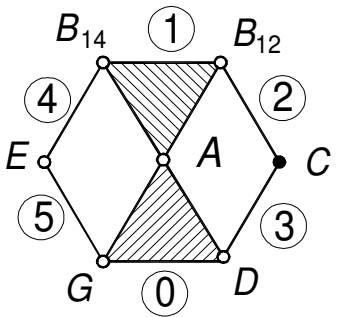

a)

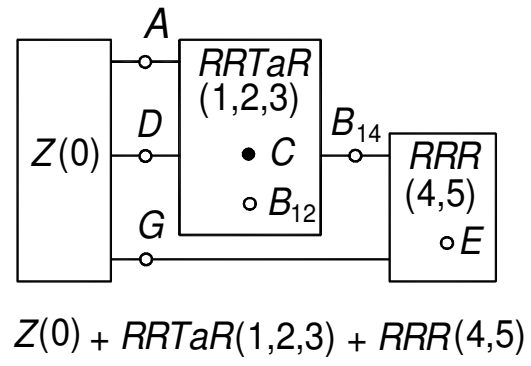

b)

Fig. 5. Structural scheme of mechanism in Figure 4 (a); multipolar scheme and structural relation of the same mechanism (b)

Figure 5 shows that the mechanism consists of the base $Z(0)$, motor dyad $R R T a R(1,2,3)$ and the dyad of aspect $1 R R R(4,5)$.

When the $B T$ platform has reached the car body level and the pallet has been unloaded, the hydraulic cylinder formed with the elements $2-3$ is blocked, and the point $B$ becomes fixed (the $A B D$ triangle is formed). For this case, the kinematic, structural and multipolar schemes of the mechanism are shown in Figure 6.

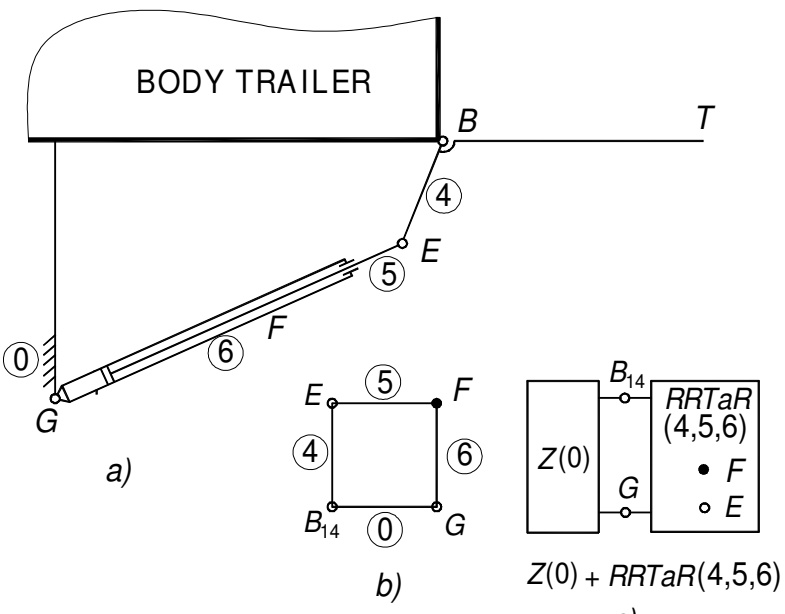

c)

Fig.6. Mechanism of lifting shutter at the beginning of folding phase: a - kinematic scheme of mechanism; $b$ - structural scheme; $\mathrm{c}$ - multipolar scheme and structural relation

If the relative movements between the elements are taken into account, it is noted that the mechanism in Figure 6 has the following lower couplings: $B_{40}(4 R 0), E(4 R 5), F(5 T 6)$ and $G(6 R 0)$.

The number of upper couplings is zero.

The movable elements of the mechanism are: $1\left(A, B_{12}, \mathrm{~B}_{14}\right), 2\left(B_{12}, C\right), 3(C, D), 4(B, E), 5(E, F)$ and $6(F, G)$. In this case, the mobility degree of the mechanism is $M=1$, namely, one independent parameter is required so that the elements of the mechanism have uniquely determined movements.

Figure 6 shows that the mechanism consists of the base $Z(0)$ and the motor dyad $R R T a R(4,5,6)$.

\section{Kinematic analysis of the mechanism}

The kinematic analysis of the mechanism consists of:

1. establishing the initial position of the mechanism;

2. drawing up the calculation program to determine the kinematic parameters of the mechanism elements while the $B T$ platform receives a circular translation movement until it reaches the body level, then a positive rotation with an angle of $\pi / 2 \mathrm{rad}$; 
3. tabular presentation of the angular values made by the vectors $\overline{A B}, \overline{D B}, \overline{B E}$ and $\overline{G E}$ with the positive direction of the $O x$ axis;

4. drawing the variation diagrams of angular velocities and accelerations of the mechanism elements, depending on the position of the mechanism.

When drawing up the calculation program, the operation of the mechanism is considered in two phases, namely: lifting the platform to the level of the body and folding the platform with an angle of $\pi / 2 \mathrm{rad}$.

\section{Results}

Using previous theoretical results, numerical simulation was done using the following values of the elements:

1. dimensions of the elements and the positions of the coupling adjacent to the base, as follows: $A B=0.700 \mathrm{~m}, B E=0.1581 \mathrm{~m}, X A=0.0 \mathrm{~m}, Y A=0.0 \mathrm{~m}, X D=0 \mathrm{~m}, Y D=-0.150 \mathrm{~m}, X G=-0.050$ $\mathrm{m}, Y G=-0.150 \mathrm{~m}, \alpha=1.89 \mathrm{rad}$;

2. initial position of the mechanism: $s 01=B D_{\text {init }}=0.600 \mathrm{~m}, G E=A B=0.700 \mathrm{~m}$ (platform lifting phase), $s 02=G E_{\text {init }}=0.700 \mathrm{~m}$ (platform folding phase);

3. relative velocities in couplings $C$ and $F: v_{23}=0.100 \mathrm{~m} \cdot \mathrm{s}^{-1}, v_{45}=0.100 \mathrm{~m} \cdot \mathrm{s}^{-}$;

4. relative accelerations in couplings $C$ and $F: a_{23}=0.0 \mathrm{~m} \cdot \mathrm{s}^{-2} ; a_{45}=0.0 \mathrm{~m} \cdot \mathrm{s}^{-2}$;

5. active strokes of the cylinders: stroke $1=0.200 \mathrm{~m}$, stroke $5=0.220 \mathrm{~m}$.

Figures 7 and 8 illustrate the kinematic schemes of the mechanism for lifting and folding the shutter, highlighting the position parameters.

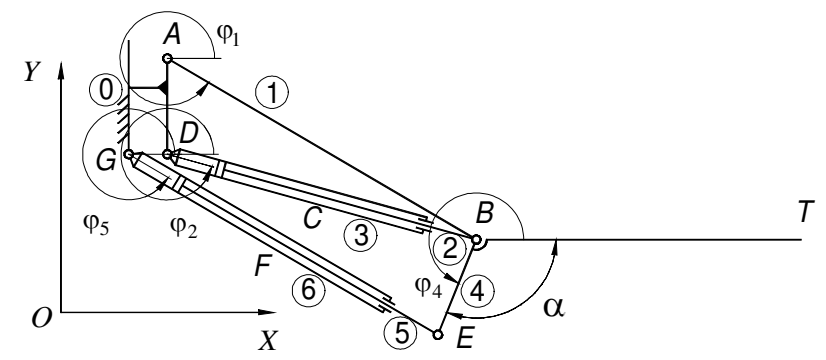

Fig. 7.Defining position kinematic parameters of mechanism (lifting phase)

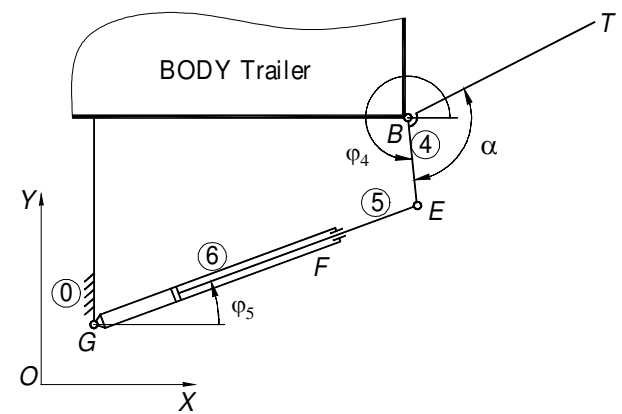

Fig. 8.Defining position kinematic parameters of mechanism (folding phase)

The calculations were made for 20 equidistant positions of the element 2 in relation to the element 3 and 23 of the equidistant positions of the element 4 in relation to the element 5 , starting from the initial position (because the original position is also considered, the total number of positions will be equal to 44).

In the lifting phase of the shutter (the $B T$ platform), the hydraulic cylinder formed by the elements 5 and 6 is blocked at a length equal to $A B$.

In the folding phase, the hydraulic cylinder formed by the elements 2 and 3 is blocked, so the point $B$ becomes a fixed point.

Table 1 presents the position parameters of the mechanism elements, both for the lifting phase of the platform and for its folding. 
Position parameters of the mechanism elements

Table 1

\begin{tabular}{|c|c|c|c|c|}
\hline Pos. & $\varphi 1$ & $\varphi 2$ & $\varphi 4$ & $\varphi 5$ \\
\hline 0 & 5.4704 & -0.6400 & 4.3906 & 5.4704 \\
\hline 1 & 5.5509 & -0.5484 & 4.3906 & 5.5509 \\
\hline 2 & 5.6271 & -0.4632 & 4.3906 & 5.6271 \\
\hline \multicolumn{5}{|c|}{$\ldots$} \\
\hline 42 & 6.9356 & 0.8020 & 5.8800 & 6.8820 \\
\hline 43 & 6.9356 & 0.8020 & 5.9568 & 6.8896 \\
\end{tabular}

Figures 9 and 10 show the variation diagrams of angular velocities and accelerations of the mechanism elements.

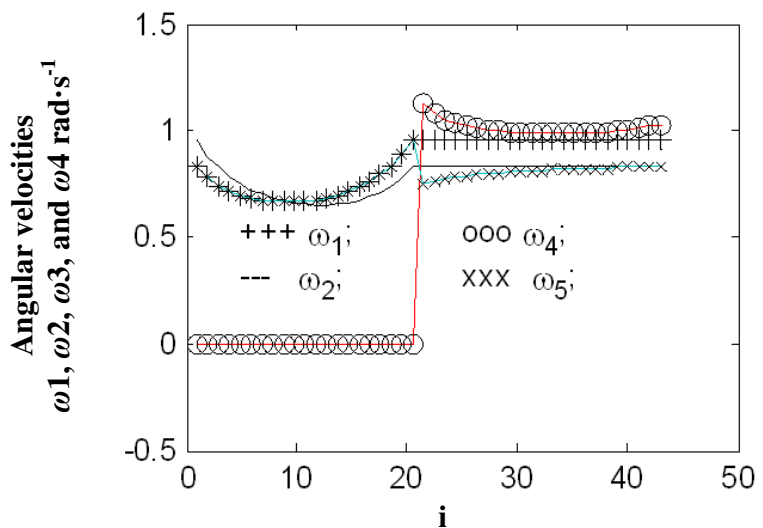

Fig. 9. Diagrams of angular velocities of mechanism elements

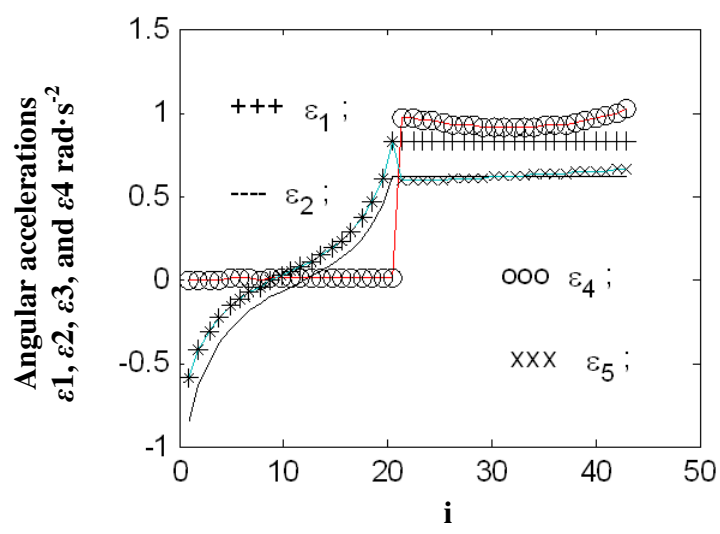

Fig. 10. Diagrams of angular accelerations of mechanism elements

\section{Conclusions}

1. The simulation performed using this mathematical model was very useful in the concrete sizing of the elements that conducted to the optimizing of the mechanism. In practice, the results were used to design the experimental model of the machine, and then for manufacturing the machine designed at INMA Bucharest.

2. A correct dimensioning of the components of the mechanism ensures silent operation of the mechanism, without great variations in angles and accelerations, this being the purpose of the research.

3. In the two operating phases of the mechanism, lifting the shutter until it reaches the platform level and ramping it until it reaches the vertical plane and the closure of the bin are established, it is found that the angular speed is constant on the two portions, which denotes a shock-free operation of the mechanism, ensuring undamaged loading of the transported goods.

4. Theoretical researches through mathematical modelling and experimental research through simulation led to a shortening of the product realization period.

5. The kinematic analysis of the mechanism precedes its kinetostatic analysis, namely, the determination of the reaction forces in the kinematic couplings and the driving force of the hydraulic cylinder, which will be done in the next research.

\section{Acknowledgement}

This work was supported by a grant of the Romanian Research and Innovation Ministry, through Programme 1 - Development of the national research-development system, subprogramme 1.2 Institutional performance - Projects for financing excellence in RDI, contract No. 16PFE, based on the research conducted on the project No.7C12/13.09.2004,"Modern equipment for road transport in a containerized and palletized system of goods, with a payload capacity of 5 tons, according to 
European standards", by AMTRANS Program, which was led by the Romanian Ministry of Research and Innovation.

\section{References}

[1] Myszka D. Machines \& Mechanisms. Applied Kinematic Analysis. $4^{\text {th }}$ edition. Prentice Hall Publishing House, 2014, 376 p.

[2] MataA.S.,Bataller T.A., CabreraC.J.A. et al.Fundamentals of Machine Theory and Mechanisms. SpringerLink, 2016,400 p.

[3] Garcia-Prada J.C., Castejón C.New Trends in Educational Activity in the Field of Mechanism and Machine Theory. SpringerLink, 2014, 310 p.

[4] Popa L. Modern equipment for road transport in a containerized and palletized system of goods, with a payload capacity of 5 tons, according to European standards (Echipament modern de transport rutierînsistemconteinerizatşi paletizat al mărfurilor, cu masa utila de 5 tone, corespunzătorstandardeloreuropene). Project No.7C12/13.09.2004, AMTRANS Programme, Romania.

[5] Artobolevski I.I. Théorie des Mécanismes et des Machines (Theory of Mechanisms and Machines). Moscow: Ed. Mir, 1977. 453 p. (In French).

[6] Demidovich B., Maron A.I. Elements of Numerical Calculation (Éléments de calculnumérique). 2nd edition. Moscow: Ed. Mir, 1987. 717 p. (In French).

[7] Dorn W.S., Mc Cracken D.D. Metodenumericeînlimbajul Fortran IV (Numerical methods with programs in FORTRAN IV). Bucharest: Technical Publishing House, 1976, 468 p. (In Romanian).

[8] Duca C., Buium Fl., ş.a. Mecanisme (Mechanisms), Iasi: GheorgheAsachi Publishing House, 2003, 481p. (In Romanian).

[9] Moise V., Maican E., Moise Şt. I. Metodenumericeîninginerie (Numerical methods in engineering) / Bucharest: Bren Publishing House, 2003, 305 p. (In Romanian).

[10] Moise V., Simionescu I., Ene M. etc. Analizamecanismeloraplicate(Analysis of Applied Mechanisms), Bucharest, Printech Publishing House, 2008, 282 p. (In Romanian).

[11] Moise V., Simionescu I., Ene M., Rotaru, Al., Analizamecanismelor plane cu bare articulate (Analysis of planar mechanisms with articulated bars), Bucharest, Printech Publishing House, 2015, 324 p. (In Romanian).

[12] Pelecudi Chr. Preciziamecanismelor(The precision of the mechanisms), Bucharest: Publishing House of the Academy of the Socialist Republic of Romania, 1975, 398 p. (In Romanian).

[13] Pelecudi Chr., Maroş D., Merticaru V. etc. Mecanisme(Mechanisms), Bucharest: Didactic and Pedagogical Publishing House, 1985, 394 p.(In Romanian).

[14] Simionescu I., Moise V. Mecanisme(Mechanisms), Bucharest: Technical Publishing House, 1999, 238 p. (In Romanian).

[15] Uicker J.J., Pennock G.R., Shigley J. E. Theory of Machines and Mechanisms. 4th Edition. New York: Oxford University Press, 2011. 928 p. 